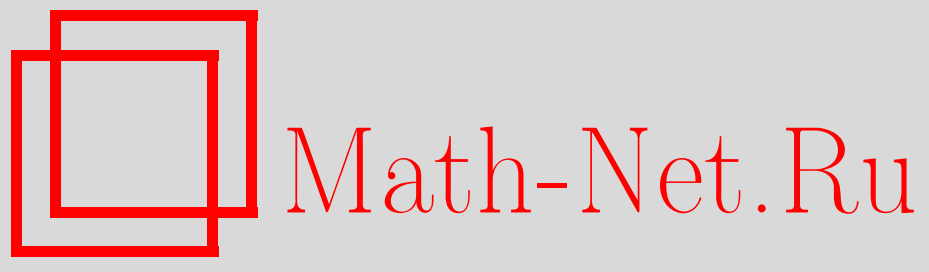

А. И. Зыкин, Асимптотические свойства дзета-функции Дедекинда в семействах числовых полей, УМH, 2009, том 64, выпуск 6, 175-176

DOI: https://doi.org/10.4213/rm9296

Использование Общероссийского математического портала Math-Net.Ru подразумевает, что вы прочитали и согласны с пользовательским соглашением http://www . mathnet.ru/rus/agreement

Параметры загрузки:

IP: 54.80 .97 .219

26 апреля 2023 г., 12:11:34

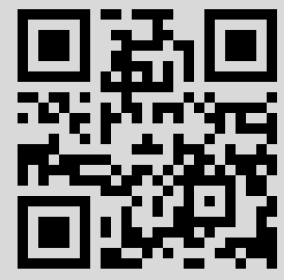




\section{Асимптотические свойства дзета-функции Дедекинда в семействах числовых полей}

\section{А. И. Зыкин}

Нашей отправной точкой является классическая теорема Брауэра-Зигеля для числовых полей, доказанная Р. Брауэром в [1]. Она утверждает, что если $K$ пробегает последовательность числовых полей, нормальных над $\mathbb{Q}$ и таких, что $n_{K} / \log \left|D_{K}\right| \rightarrow 0$, то $\log \left(h_{K} R_{K}\right) / \log \sqrt{\left|D_{K}\right|} \rightarrow 1$. Здесь $D_{K}, h_{K}$ и $R_{K}$ - дискриминант, число классов и регулятор поля $K$ соответственно.

Эта теорема была обобщена М. А. Цфасманом и С. Влэдуцем в [2] на случай, когда условие $n_{K} / \log \left|D_{K}\right| \rightarrow 0$ не выполнено. Для того чтобы сформулировать этот результат, нам понадобится несколько обозначений. Для конечного расширения $K / \mathbb{Q}$ положим $\Phi_{q}(K)$ - число простых идеалов кольца целых $\mathscr{O}_{K}$ с нормой $q$, кроме того, обозначим $\Phi_{\mathbb{R}}(K)$ и $\Phi_{\mathbb{C}}(K)$ число вещественных и комплексных вложений $K$ в $\mathbb{C}$ соответственно. Обозначим через $g_{K}=\log \sqrt{\left|D_{K}\right|}$ род поля $K$ (по аналогии со случаем кривых над конечным полем). Расширение $K / \mathbb{Q}$ называется почти нормальным, если можно найти промежуточные расширения $K=K_{n} \supseteq K_{n-1} \supseteq \ldots \supseteq K_{1} \supseteq K_{0}=\mathbb{Q}$ такие, что $K_{i} / K_{i-1}$ нормально для всех $i$.

Рассмотрим семейство попарно неизоморфных числовых полей $\left\{K_{i}\right\}$. Определим $\phi_{\alpha}=\lim _{i \rightarrow \infty} \Phi_{\alpha}\left(K_{i}\right) / g_{K_{i}}, \alpha \in\{\mathbb{R}, \mathbb{C}, 2,3,4,5,7,9, \ldots\}$. Если пределы $\phi_{\alpha}$ существуют, то семейство называется асимптотически точным. Оно называется асимптотически хорошим, если существует $\phi_{\alpha} \neq 0$, и асимптотически плохим в противном случае.

Несложно проверить, что условие $n_{K} / \log \left|D_{K}\right| \rightarrow 0$ из теоремы Брауэра-Зигеля эквивалентно тому, что соответствующее семейство асимптотически плохое. Теперь мы можем сформулировать теорему Цфасмана-Влэдуца, доказанную в работе [2] в асимптотически хорошем случае и в работе [3] в асимптотически плохом.

Теорема 1. Для асимптотически точного семейства $\left\{K_{i}\right\}$ имеет место формула:

$$
\lim _{i \rightarrow \infty} \frac{\log \left(h_{K_{i}} R_{K_{i}}\right)}{g_{K_{i}}}=1+\sum_{q} \phi_{q} \log \frac{q}{q-1}-\phi_{\mathbb{R}} \log 2-\phi_{\mathbb{C}} \log (2 \pi)
$$

при условии, что все $K_{i}$ почти нормальны над $\mathbb{Q}$ или выполнена обобщенная гипотеза Римана $(G R H)$ для дзета-функиий Дедекинда полей $K_{i}$.

Определим предельную дзета-функцию асимптотически точного семейства числовых полей:

$$
\zeta_{\left\{K_{i}\right\}}(s)=\prod_{q}\left(1-q^{-s}\right)^{-\phi_{q}} .
$$

Теорема С из [2] дает нам абсолютную сходимость бесконечного произведения при $\operatorname{Re} s \geqslant 1$. Если $\varkappa_{K}=\operatorname{Res}_{s=1} \zeta_{K}(s)$ - вычет дзета-функции Дедекинда поля $K$ в точке $s=1$, то равенство (1) можно переписать в виде $\lim _{i \rightarrow \infty} \log \varkappa_{K_{i}} / g_{K_{i}}=\log \zeta_{\left\{K_{i}\right\}}(1)$. Кроме того, в работе [2] доказано, что в области $\operatorname{Re} s>1$ имеет место формула $\lim _{i \rightarrow \infty} \log \zeta_{K_{i}}(s) / g_{K_{i}}=\log \zeta_{\left\{K_{i}\right\}}(s)$.

Основной нашей целью является изучение вопроса о наличии подобного равенства при $\operatorname{Re} s<1$. Уже случай $s=1$ по сути эквивалентен теореме Брауэра-Зигеля и имеющимися на данный момент методами не может быть рассмотрен в полной общности без предположения GRH. В дальнейшем мы предполагаем, что для всех полей, которые мы рассматриваем, для соответствующей дзета-функции Дедекинда выполнена гипотеза Римана.

Работа выполнена при поддержке РФФИ (гранты 06-01-72004-МНТИа, 06-01-72550НЦНИЛа, 07-01-00051a, 08-07-92495-НЦНИЛа) и INTAS (грант 05-1000008-8118). 
В предположении GRH в [2; следствие из теоремы A] доказано, что бесконечное произведение для $\zeta_{\left\{K_{i}\right\}}(s)$ абсолютно сходится при $\operatorname{Re} s \geqslant 1 / 2$. Теперь сформулируем наши основные результаты.

Tеорема 2. В предположении GRH при $\operatorname{Re} s>1 / 2$ для асимптотически точного семейства $\left\{K_{i}\right\}$ имеет место равенство $\lim _{i \rightarrow \infty} \log \zeta_{K_{i}}(s) / g_{K_{i}}=\log \zeta_{\left\{K_{i}\right\}}(s)$.

Доказательство теоремы использует оценки логарифмической производной дзетафункций в критической полосе совместно с теоремой Витали о предельном переходе в последовательности голоморфных функций.

При $s=1 / 2$ наш результат несколько слабее. Мы доказываем следующую верхнюю оценку.

Теорема 3. Пусть $\rho_{K_{i}}-$ первый ненулевой коэффициент в разложении $\zeta_{K_{i}}(s)$ в ряд Тейлора в точке $s=1 / 2$, т.е. $\zeta_{K_{i}}(s)=\rho_{K_{i}}(s-1 / 2)^{r_{K_{i}}}+o\left((s-1 / 2)^{r_{K_{i}}}\right)$. Тогда, в предположении GRH, для асимптотически точного семейства $\left\{K_{i}\right\}$ выполнено неравенство $\lim _{i \rightarrow \infty} \log \left|\rho_{K_{i}}\right| / g_{K_{i}} \leqslant \log \zeta_{\left\{K_{i}\right\}}(1 / 2)$.

Для доказательства теоремы 3 мы используем методы, с помощью которых получается верхняя оценка в равенстве из теоремы 1 , а также информацию о предельном распределении нулей дзета-функций на критической прямой в семействах числовых полей. Вопрос о наличии равенства в утверждении теоремы 3 весьма тонок и связан с низко лежащими нулями дзета-функций, т. е. с нулями $\zeta_{K}(s)$, имеющими маленькую мнимую часть по сравнению с $g_{K}$. Мы полагаем, что равенство $\lim _{i \rightarrow \infty} \log \left|\rho_{K_{i}}\right| / g_{K_{i}}=\log \zeta_{\left\{K_{i}\right\}}(1 / 2)$ может быть неверно для некоторых семейств $\left\{K_{i}\right\}$, ввиду "случайного" поведения низко лежащих нулей дзета-функций. Однако оно может быть верным для "большинства" семейств. Более подробное обсуждение вопроса, хотя и в несколько другой ситуации (низко лежащие нули $L$-функций, ассоциированных с модулярными формами на $\left.\mathrm{SL}_{2}(\mathbb{R})\right)$, можно найти в работе [4].

Сформулируем одно следствие из теоремы 2. Напомним, что постоянная ЭйлераКронекера числового поля $K$ определяется следующим образом $\gamma_{K}=c_{0}(K) / c_{-1}(K)$, где $\zeta_{K}(s)=c_{-1}(K)(s-1)^{-1}+c_{0}(K)+O(s-1)$. Я. Ихара в работе [5] получил асимптотическую формулу для $\gamma$ в семействах кривых над конечными полями. Мы же имеем следующий результат, вытекающий из теоремы 2.

СледСтвиЕ 4. B предположении GRH для асимптотически точного семейства числовых полей $\left\{K_{i}\right\}$ имеет место формула $\lim _{i \rightarrow \infty} \gamma_{K_{i}} / g_{K_{i}}=-\sum_{q} \phi_{q} \log q /(q-1)$.

Это утверждение сформулировано в [6] без предположения GRH. K сожалению, доказательство, данное там, содержит лакуны. Оно использует необоснованную перестановку предельных переходов в суммировании по степеням простых чисел и в пределе по семейству $\left\{K_{i}\right\}$. Таким образом, вопрос о наличии равенства без предположения GRH остается открытым.

Я выражаю благодарность моему научному руководителю М. А. Цфасману за постоянное внимание к работе, а также М. Балазару за ценные советы и идеи.

\section{Список литературы}

[1] R. Brauer, Amer. J. Math., 69:2 (1947), 243-250. [2] M. A. Tsfasman, S. G. Vlăduţ, Mosc. Math. J., 2:2 (2002), 329-402. [3] A. Zykin, Mosc. Math. J., 5:4 (2005), 961-968. [4] H. Iwaniec, W. Luo, P. Sarnak, Inst. Hautes Études Sci. Publ. Math., 91 (2000), 55-131. [5] Y. Ihara, Algebraic geometry and number theory, Progr. Math., 253, Birkhäuser, Boston, MA, 2006, 407-451. [6] M. A. Tsfasman, Algebraic geometry and number theory, Progr. Math., 253, Birkhäuser, Boston, MA, 2006, 453-458.

\section{А. И. Зыкин (А. I. Zykin)}

Математический институт им. В. А. Стеклова РАН;

Независимый московский университет

E-mail: alzykin@gmail.com
Представлено А. Б. Сосинским Принято редколлегией 05.04.2009 